\title{
FUSION OF BRUSHLET AND WAVELET DENOISING METHODS FOR NUCLEAR IMAGES
}

\author{
E. Angelini, Y. Jin, P. Esser, R. Van Heertum, A. Laine \\ Columbia University, New York, USA
}

\begin{abstract}
This paper presents preliminary results on the fusion of denoised PET and SPECT data volumes from brushlet and wavelet thresholding methods. Texture-based brushlet denoising is well suited for enhancement of physiological information while wavelet-based denoising is better suited for enhancement of anatomical contours. A three-dimensional multi-scale edge-based data fusion algorithm is applied to combine enhanced data from these two independent denoising methods. Preliminary results with qualitative evaluation of PET and SPECT data by an expert clinician showed great potential of this approach to combine enhancement of both anatomical and physiological signal information for improved image quality.
\end{abstract}

\section{INTRODUCTION}

De-noising of SPECT and PET images is a challenging task due to the inherent low signal-to-noise ratio of the acquired data. The majority of clinical reading platforms apply low-pass filtering and averaging operators as preprocessing to eliminate noise components and improve the visual quality before diagnostic interpretation of the data.

In this work we fuse two distinct multiscale denoising methods. A first approach is based on wavelet expansion and thresholding of sub-band coefficients for elimination of the noisy components in spatial-frequency space prior to reconstruction. In the case of high noise levels, detailed scales of sub-band images are usually dominated by noisy components that are not well handled using traditional thresholding schemes. To address this issue, a cross-scale regularization scheme was introduced in [1], which takes into account across scale coherence of structured signals in wavelet coefficient subbands. Preliminary results showed promising performance in denoising clinical SPECT and PET images for liver and brain studies.

Our second denoising method is based on texture analysis of the images with brushlet expansion [2]. PET and SPECT images reconstructed using 2D filtered backprojection (FBP) are often accompanied with highly structured textural noise in the background. Brushlet functions offer an orthogonal framework for expansion of the images and thresholding of coefficients with high energy that correspond to the noisy textural components. We previously presented preliminary results on PET and SPECT clinical data using this approach [3]. In this study we present initial results from the fusion of these two denoising methods into a single representation of the data. We followed a feature-based fusion technique performed in a wavelet transform domain to combine edge information from the two denoised volume data sets. In our application to PET and SPECT data, fusion of the images was performed to improve the overall denoising performance for visual inspection. We therefore chose to evaluate the potential improvement of data quality by presentation of the data to an expert clinician for evaluation of the different denoising methods as relevant for improving his diagnosis tool. Results presented in this paper are very encouraging and show significant improvement of image quality and informative value after fusion.

\section{METHOD}

\subsection{Dyadic Wavelet Expansion}

Given a signal $f(x)$ a wavelet transform of the signal at scale $s$ with translation $u$ is defined by:

$$
W f(u, s)=f^{*} y_{u, s}=\grave{\mathrm{O}} f(x) \frac{1}{\sqrt{s}} y^{*}\left(\frac{x-u}{s}\right) d x \text {. }
$$

A discrete wavelet transform can be obtained by sampling the dilation parameter with a power of two and the translation parameter by a multiple of the dilation step. Sampling the translation parameter with the same resolution as the input signal, as proposed by Mallat and Zhong [4] results in a translation-invariant expansion. In that context, a dyadic 'overcomplete' wavelet transform of a signal $f(x)$ is defined as a sequence of functions

$$
\begin{aligned}
& \left\{W_{m} f(x)\right\}_{m \hat{1} Z} \text {, where } \\
& \qquad W_{m} f(x)=f * y_{m}(x),
\end{aligned}
$$

with $y_{m}(x)=2^{-m} y\left(2^{-m} x\right)$ the wavelet function $y(x)$ expanded by a dilation parameter (or scale) $2^{m}$. 
In the present work, a 3D dyadic overcomplete wavelet expansion is performed on volume image data sets using a set of three wavelet functions $\left(y^{1}, y^{2}, y^{3}\right)$ derived as the partial derivatives of a 3D smoothing function $q(x, y, z)$. In this work, we used the first derivative of a cubic spline function as the wavelet analysis basis. This type of wavelet function, illustrated in Figure 1 is well suited for edge based feature analysis.

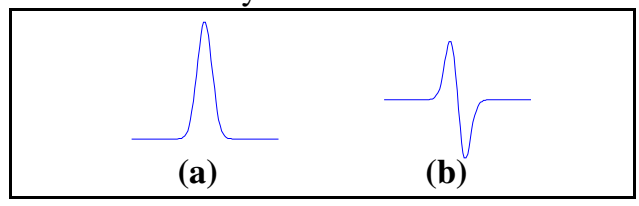

Figure 1: (a) Cubic spline smoothing function $\theta(x)$. (b) Quadratic spline wavelet $\psi(x)$ of compact support defined as the first derivative of the smoothing function $\theta(x)$.

The dyadic wavelet transform of a volume image $I(x, y, z)$ at a scale $2^{j}$ has therefore four components:

$$
T_{j}^{k} I(l, m, n)=<I, y_{j, l, m, n}^{k}>,(k=1,2,3),
$$

and a DC component, which represents the "residual" information or average energy distribution. Such a discrete dyadic wavelet transform is efficiently implemented with a hierarchical filtering scheme.

\subsection{Brushlet Expansion}

A parallel approach investigated by our group in a previous study on SPECT brain data used a brushlet multiscale expansion to characterize texture noise components in 2D slices [3]. The family of waveletpacket like functions called brushlets is constructed on sub-intervals of the real axis $\hat{c}_{n} a_{n+1} a_{\text {ù }}$ with windowed complex exponential functions as:

$$
\begin{aligned}
u_{j, n}(x)= & b_{n}\left(x-c_{n}\right) e_{j, n}(x)+v\left(x-a_{n}\right) e_{j, n}\left(2 a_{n}-x\right) \\
& -v\left(x-a_{n+1}\right) e_{j, n}\left(2 a_{n+1}-x\right)
\end{aligned}
$$

where $c_{n}=\left(a_{n}+a_{n+1}\right) / 2, b_{n}$ and $v$ are two windowing funtions, and $e_{j, n}$ is the complex value exponential function defined as: $e_{j, n}(x)=1 / \sqrt{l_{n}} e^{-2 i p j \frac{\left(x-a_{n}\right)}{l_{n}}}$. The windowing functions $b_{n}$ and $v$ and the basis function $u_{j, n}$ are displayed in Figure 2.

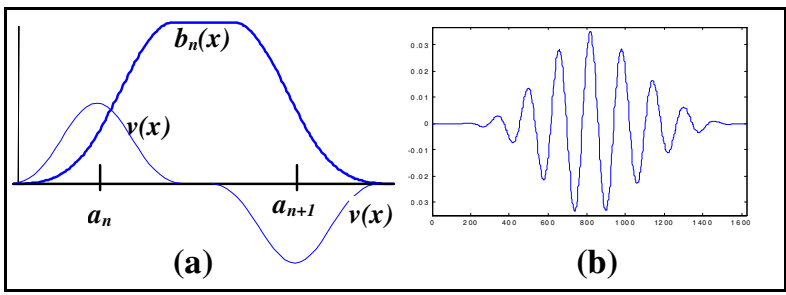

Figure 2: Windowing functions for the definition of a brushlet function on a subinterval of the real axis.

The series of functions $\left\{u_{n, j}\right\}_{n, j \hat{\imath} \notin}$ constitutes an orthonormal basis of the real axis with arbitrary tiling (i.e. partitioning into sub-intervals). In $\mathrm{N}$-dimensions, brushlet basis functions decompose a signal along specific spatial directions via analysis of its Fourier domain. In this study, a brushlet expansion was performed in 2D instead of $3 \mathrm{D}$ as the background texture is not continuous in 3D space and could therefore not be well characterized in terms of texture components with 3D brushlet functions. Similarly to the shift-invariant wavelet dyadic expansion, brushlet expansion was also performed in an overcomplete framework as described in [5].

\subsection{Denoising via Thresholding}

Denoising of a given signal using wavelet or brushlet expansions is performed via thresholding of expansion coefficients prior to reconstruction.

In the case of a wavelet expansion, coefficients with larger magnitudes are related to salient edge features in the data, showing high-correlation with the analysis function. A soft thresholding (wavelet shrinkage) operator is used. Because $\left(y^{1}, y^{2}, y^{3}\right)$ are partial derivatives of a single smoothing function $q$, the three components are proportional to the three coordinate components of the gradient vector of $I(x, y, z)$ smoothed by a dilated version of $q(x, y, z)$. From these components, one can compute the magnitude of the smoothed signal variation, which is proportional to the wavelet coefficient modulus:

$$
M F=\sqrt{\left|T_{j}^{1} F\right|^{2}+\left|T_{j}^{2} F\right|^{2}+\left|T_{j}^{3} F\right|^{2}} .
$$

In the proposed method, a threshold operator was applied to these wavelet coefficients modulus which are equivalent to edge maps at different scales. Traditional thresholding level selection schemes perform poorly on very noisy textured data such as PET and SPECT volumes. Indeed detailed levels of wavelet sub-band images display high-energy coefficients for both edge features and noise components. A cross-scale regularization scheme was used to guide the threshold level selection from one expansion level to the previous as detailed in [1]. This regularization scheme, which brings spatial adaptivity to the thresholding operator, was motivated by the idea that meaningful signal features 
show persistent higher levels of coherence across wavelet expansion scales when compared to random noise. The DC component, which contains most of the energy of the projected signal, was preserved in the thresholding procedure.

Regarding thresholding of the brushlet expansion we adopted a spatially adaptive thresholding level scheme initially suggested by Chang et al. [6] and applied by our group in [3]. This approach identifies in the noisy image three regions for edges (using a Canny edge detector), smooth background (based on the level of the local variance in the signal) and texture areas (defined as nonedges and non-smooth areas). A hard thresholding operator was applied to the coefficients with different threshold levels for each of the three regions. The threshold level was set proportional to a minimax global level, derived from the noise variance in the image using a weight of 0.25 on edges and texture areas and 0.5 in smooth areas.

Comparing the two denoising methods on clinical PET and SPECT data, we observed in our previous experiments a better performance of brushlet denoising at removing background noise and enhancing physiological information (with higher details of brightness levels within distinct anatomical structures) than with wavelet denoising but a poorer spatial delineation of anatomical contours. This observation thus motivated the development of a fusion algorithm to combine the two denoising methods.

\subsection{Fusion of the Two Denoising Methods}

Fusion deals with the problem of combining different or incomplete representations of the same object into a single representation that integrates the different sources and pieces of information. Several wavelet-based techniques have been proposed to perform fusion based on localization of salient features in individual sub-band representations $[7,8]$.

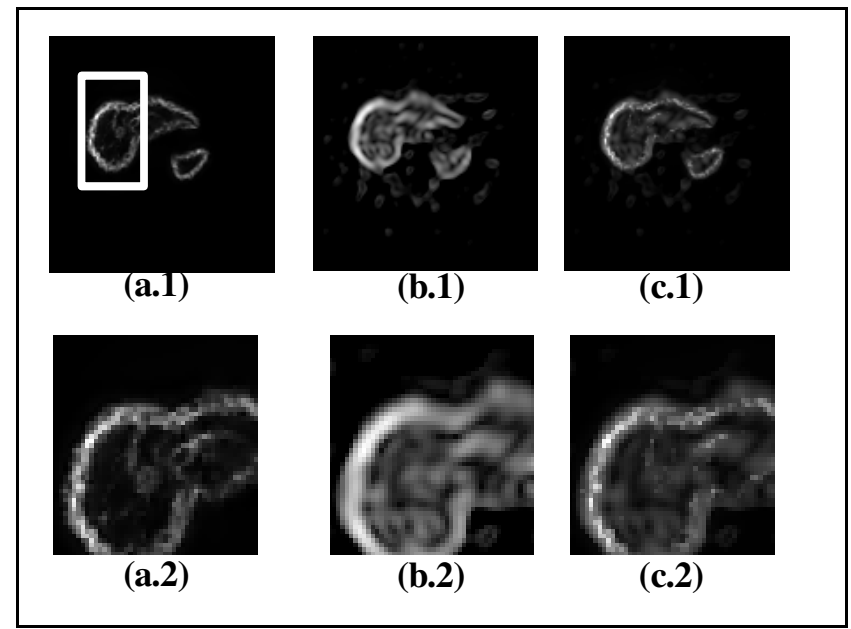

Figure 3: Fusion of wavelet coefficient modulus in first level of expansion. Coefficient modulus maps are displayed for denoised data with: (a) wavelet thresholding, (b) brushlet thresholding, (c) fused coefficients. Coefficient modulus maps are displayed in (a.1, b.1, c.1) for a cross-slice from a SPECT liver data, and (a.2, b.2, c.2) for a ROI within a slice.

In this framework, the images to fuse are expanded on a selected set of wavelet basis functions, a fusion rule is applied on the wavelet coefficients in the transform domain and a single data representation is reconstructed with the fused wavelet coefficients. Nikolov et al. proposed in [7] a fusion of 2D images using the same wavelet expansion framework as applied in this work for denoising, based on wavelet coefficients modulus. We followed the same general approach, but expanding the fusion rule in three dimensions: Given two volume data sets denoised with wavelet and brushlet thresholding, we expand the volumes onto the same wavelet expansion as used for denoising (based on a spline first derivative analysis function). Coefficient modulus maps are computed for the two volumes and the fusion rule is applied that extracts the maximum modulus of the two maps. The corresponding coefficients are used for reconstruction of a fused representation of the two denoised volumes. We point out here that using denoised data for fusion provided representations with sparse edge information and eliminated the need to empirically set a threshold level in the fusion rule as performed in [7]. We illustrate in Figure 3 this fusion process on the first subband coefficient modulus maps of a liver SPECT data set.

\section{RESULTS}

\subsection{Experimental Setup}

We present in this section results on denoising and fusion of PET and SPECT clinical data sets reconstructed with filtered back projection (FBP). A 3D wavelet expansion was performed for denoising and fusion using three analysis levels. Brushlet denoising was performed with a [8×8] tiling of the Fourier plane in $2 \mathrm{D}$.

\subsection{Denoising Results}

We illustrate in the Figures 4 and 5 the denoising performance of the two methods on a liver SPECT data set and a brain PET data set. 


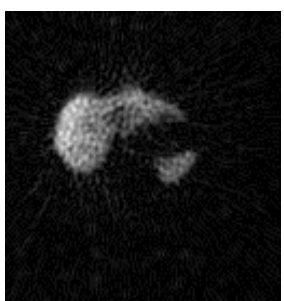

(a)

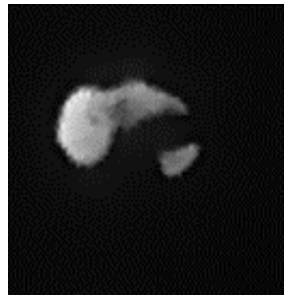

(c)

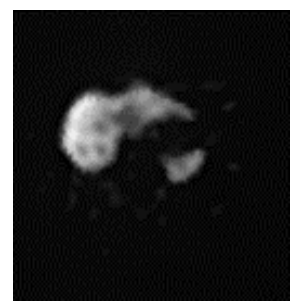

(b)

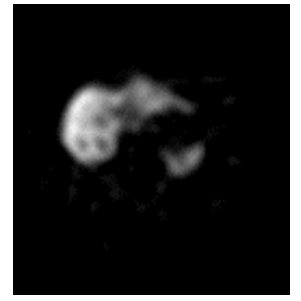

(d)
Figure 4: (a) Clinical SPECT liver data set reconstructed using FBP with clinical settings. (b) Fusion of the two denoising methods: (c) brushlet thresholding and (d) wavelet thresholding.

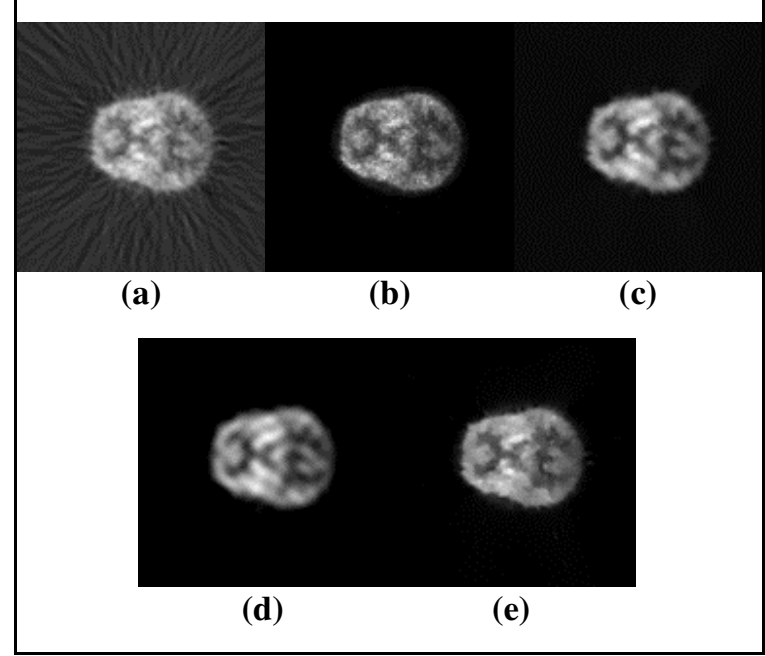

Figure 5: (a) Clinical PET brain exam reconstructed using FBP with clinical settings. (b) Image reconstructed using OSEM with clinical settings for brain data. (c) Fusion of the two denoising methods: (d) brushlet thresholding and (e) wavelet thresholding.

We also show the results of the fusion of the two denoising methods. For both experiments, visual inspection of the results showed a clear improvement of image quality with both denoising methods compared to simple FBP.

\subsection{Evaluation of Image Quality by a Clinician}

An experienced clinician specialized in nuclear medicine participated in a review session for qualitative evaluation of the denoising methods. The clinician was presented with the denoised 3D data sets from (1) wavelet thresholding, (2) brushlet thresholding and (3) fused denoised results. The visualization platform allowed him to compare simultaneously the three volumetric data sets on a single GUI, slice by slice. The clinician was then asked to compare the fused data set to a control data set reconstructed with clinical settings without any postprocessing. For both clinical cases, the clinician observed clear improvements in image contrast and the definition of physiological activities inside the target organs for the denoised images. Denoising with brushlet thresholding was considered beneficial for enhancing "internal edges", e.g. anatomical or physiological variations within the target organs. Wavelet modulus analysis using cross-scale regularization was recognized beneficial for enhancing "external edges", with a better definition and delineation of the organ contours. The clinician was also confident that the fused images effectively combined important features from both enhanced images, without introducing artifacts.

For the PET brain data set, we also asked the clinician to compare the results from our denoising scheme and the reconstruction with the ordered subset expectation maximization (OSEM) method, considered the state-ofthe-art for tomographic reconstruction. The conclusion of the qualitative comparison was that the fused multi-scale denoising methods provided significantly improved image quality in terms of both lower noise level and better contrast for key anatomical and physiological features.

\section{CONCLUSION}

We proposed in this paper results from a preliminary study on PET and SPECT data to evaluate new applications for fusion of denoising methods using a wavelet-based approach. Wavelet expansion provides a feature-based fusion method, which combines sparse edge representations of the denoised images into multiscale edges via a fusion rule that selects predominant wavelet coefficient modulus. Results showed an efficient fusion of the denoised methods with data sets that offered a significant enhancement of both anatomical and physiological data. Full evaluation of the denoising methods and merit of the fusion approach will require a phantom study with exact knowledge of the shapes and activity levels, followed by a clinical study on data sets with pathological ground truth.

\section{REFERENCES}

[1] Y. Jin, E. Angelini, P. Esser, and A. Laine, "De-noising SPECT/PET images using cross-scale regularization," MICCAI, pp. 32-40, Montreal, Canada, 2003.

[2] F. Meyer and R. R. Coifman, "Brushlets: A tool for directional image analysis and image compression," Applied and Computational Harmonic Analysis, vol. 4, No. 1, pp. 147-187, 1997.

[3] E. D. Angelini, J. Kalifa, and A. F. Laine, "Harmonic multiresolution estimators for denoising and regularization 
of SPECT-PET data,"IEEE International Symposium on Biomedical Imaging, pp. 697-700, Washington, D.C., USA, 2002.

[4] S. Mallat and S. Zhong, "Signal characterization from multiscale edges," 10th International Conference on Pattern Recognition, pp. 891-896, Atlantic City, NJ, USA, 1990.

[5] E. Angelini, A. Laine, S. Takuma, J. Holmes, and S. Homma, "LV volume quantification via spatio-temporal analysis of real-time 3D echocardiography," IEEE Transactions on Medical Imaging, vol. 20, No. 6, pp. 457469, 2001.

[6] S. G. Chang, B. Yu, and M. Vetterli, "Spatially adaptive wavelet thresholding with context modeling for image denoising," IEEE International Conference on Image Processing, pp. 535 -539, Chicago, IL, USA, 1998.

[7] S. G. Nikolov, D. R. Bull, C. N. Canagarajah, M. Halliwell, and P. N. T. Wells, "Fusion of 2-D images using their multiscale edges, "IAPR International Conference on Pattern Recognition, pp. 41-44, Barcelona, Spain, 2000.

[8] I. Koren, A. Laine, and F. Taylor, "Image fusion using steerable dyadic wavelet transform," IEEE International Conference on Image Processing, pp. 232-235, Washington, D.C., USA, 1995. 